\title{
Congenital left internal thoracic artery-pulmonary artery fistula used as an inflow for a coronary artery bypass graft
}

\author{
Yoshiharu Soga, MD, PhD, ${ }^{a}$ Michiya Hanyu, MD, PhD, ${ }^{a}$ Masahide Kawatou, MD, ${ }^{\text {a }}$ Tadaaki Yokota, MD, PhD, \\ Takuya Nomoto, MD, PhD, ${ }^{a}$ Jota Nakano, MD, ${ }^{a}$ and Hitoshi Okabayashi, MD, PhD, ${ }^{a}$ Kitakyushu, Japan
}

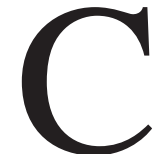

ongenital left internal thoracic artery (LITA)-pulmonary artery (PA) fistula is rare. We describe such a case in which the fistula was used as an inflow for a coronary artery bypass graft in a patient with diffuse calcification of the ascending aorta.

\section{Clinical Summary}

An 81-year-old woman with left main trunk and triple-vessel coronary artery disease was referred to our hospital for surgical treatment. A LITA-PA fistula had also been diagnosed by means of angiography preoperatively.

The patient underwent off-pump coronary artery bypass grafting in July 2003. Because preoperative computed tomographic (CT) analysis showed a severely calcified ascending aorta, we had prepared an automatic proximal anastomosis device for a saphenous vein graft (SVG). After a median sternotomy, the LITA-PA fistula was easily identified and was found to have diverged at the level of the second intercostal space (Figure 1, A). An ultrasonographic scalpel was used for harvesting both the LITA and the fistula. The proximal fistula had almost the same diameter as the LITA. Calcified lesions were scattered diffusely in the wall of the ascending aorta, as detected by means of CT preoperatively. In addition, because the caliber of the SVG was relatively small $(3.5-4.0 \mathrm{~mm})$, we decided to use the fistula as an inflow for the SVG to avoid aortic manipulation. First, the SVG was anastomosed with 8-0 Prolene sutures (Ethicon, Somerville, NJ) in an end-to-end manner to make a composite $\mathrm{Y}$ graft (Figure 1,B). Then, the LITA was anastomosed to the left anterior descending coronary artery, the SVG to the circumflex coronary artery, and the gastroepiploic artery to the right coronary artery. After completion of the bypass grafting, transit-time flow measurement was performed, and this revealed a good flow volume and diastolic dominant pattern in all 3 grafts.

Angiographic analysis on the seventh postoperative day showed that all the grafts were patent. The patient was discharged uneventfully and is currently in good clinical condition, being followed up at our outpatient clinic. Midterm graft evaluation was done by using

From the Departments of Cardiovascular Surgery ${ }^{\mathrm{a}}$ and Pathology, ${ }^{\mathrm{b}}$ Kokura Memorial Hospital, Kitakyushu, Japan.

Received for publication June 19, 2007; accepted for publication July 5, 2007.

Address for reprints: Yoshiharu Soga, MD, PhD, Department of Cardiovascular Surgery, Kokura Memorial Hospital, 1-1, Kifune-machi, Kokurakita-ku, Kitakyushu, 802-8555, Japan (E-mail: sogakin@dd.iij4u.or.jp).

J Thorac Cardiovasc Surg 2007;134:1581-2

$0022-5223 / \$ 32.00$

Copyright (C) 2007 by The American Association for Thoracic Surgery doi:10.1016/j.jtcvs.2007.07.055 multirow detector CT in October 2006, and this revealed total graft patency (Figure 1,C).

\section{Discussion}

LITA-PA fistulae are reported to be associated with a number of different pathologies, but most of them develop after coronary artery bypass surgery. LITA-PA fistula is known to cause recurrent angina and LITA bypass occlusion. ${ }^{1-3}$ In contrast, a congenital LITA-PA fistula is rare, but angiography of the LITA before surgical intervention is generally not a standard procedure, and as a result, the presence of a congenital fistula might be overlooked. ${ }^{2}$

In this report, we have described, for the first time, that a congenital LITA-PA fistula can be used as an inflow for the SVG and that the SVG can remain patent for more than 3 years after surgical intervention. Although recognizing that the fistula in this case was usable in view of its appropriate location and size, it is significant in that it allowed any surgical manipulation of the aorta to be avoided because the patient was more than 80 years old and had a diffusely calcified aorta that could not be clamped. As a result, no complications occurred.

Late patency still remains a concern in patients with coronary artery bypass grafts. Histologic analysis of the LITA remnant and the fistula obtained from this patient, as shown in Figure 2, revealed that the fistula had an artery-like structure like the LITA, but its intima had partial fibrous hyperplasia and was thicker than that of the LITA. Although it is unknown whether histologic characteristics affect late graft patency, aneurysm formation in a LITA-PA fistula has been reported. ${ }^{4,5}$ Therefore, this patient will require careful follow-up.

In conclusion, a LITA-PA fistula can be used as an inflow for a coronary artery bypass graft, yielding satisfactory early patency, and might be useful in patients with a calcified aorta.

\section{References}

1. Garrean S, Tshibaka C, Hanhan Z, Geha AS, Massad MG. Coronarypulmonary steal caused by internal thoracic artery-pulmonary artery fistula after coronary artery bypass operations. J Thorac Cardiovasc Surg. 2005;130:569-71.

2. Riess FC, Lehmann C, Loewer C, Schofer J, Bleese N. Congenital fistula between the left internal mammary artery (LIMA) and the pulmonary artery: cause of LIMA bypass occlusion? Heart Surg Forum. 2004; 7:E403-4.

3. Hearne SF, Burbank MK. Internal mammary artery-to-pulmonary artery fistulas. Case report and review of the literature. Circulation. 1980;62: 1131-5.

4. Den OG, Stam J. Aneurysm of internal mammary artery. Thorax. 1978;33:526-7.

5. Hachiro Y, Sugimoto S, Takagi N, Abe T. Aneurysmal change in an internal mammary artery-pulmonary artery fistula. Ann Thorac Surg. 2001;72:1377-8. 


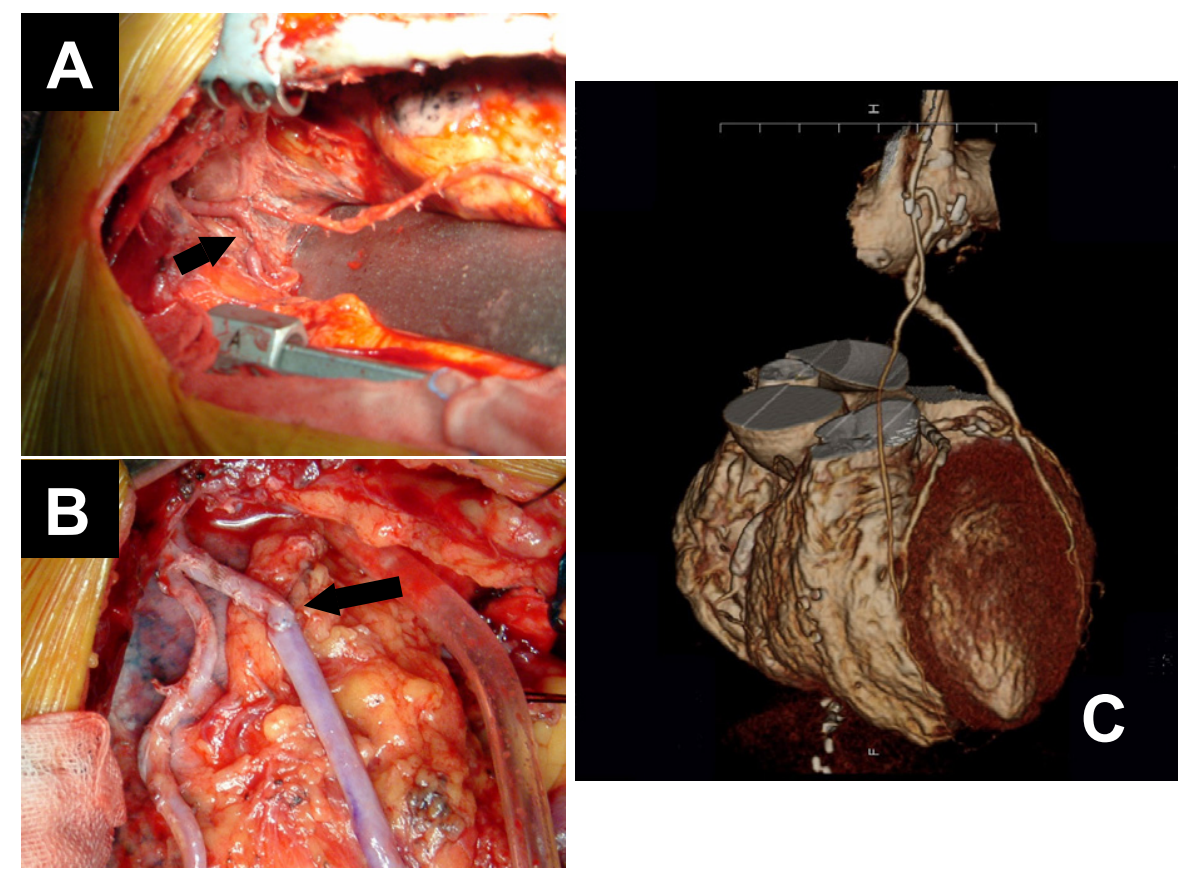

Figure 1. Representative photographs of the left internal thoracic arterypulmonary artery fistula (arrow) (A), composite $Y$ graft in which the saphenous vein graft was anastomosed with the proximal fistula in an end-to-end manner (arrow indicates anastomosis site) (B), and multirow detector CT scan performed 3 years after the operation (C).
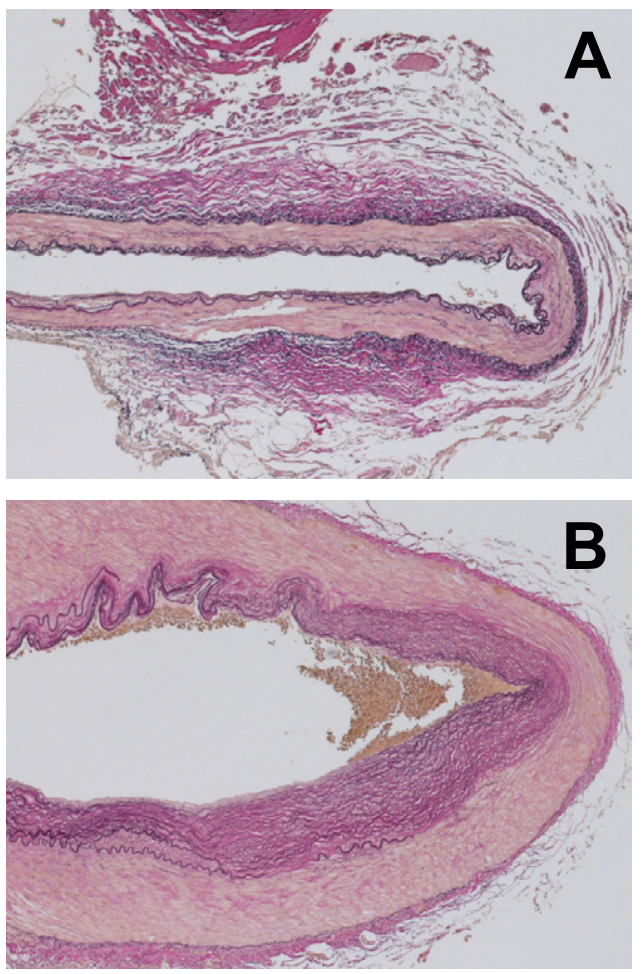

Figure 2. Representative photographs of the internal thoracic artery $(A)$ and fistula $(B)$ stained with elastica van Gieson. 